\title{
Errata
}

\section{Probabilistic Kinetics of}

Macroprocesses in Broken

Microscopic Reversibility

Koichiro Matsuno ${ }^{2}$

Expressions (52) and (55) should read

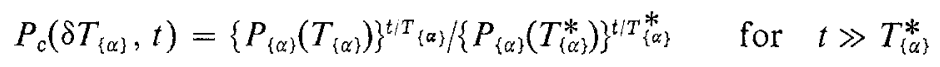

and

$$
\delta T_{\{\alpha\}} \leqslant 0
$$

Accordingly, the resulting expressions should be corrected as follows:

$$
\begin{aligned}
\left\langle\delta T_{\{\alpha\}}^{2}\right\rangle_{t} & =\frac{2\left(T_{\{\alpha\}}^{*}\right)^{2}}{\left[\ln P_{\{\alpha\}}^{*}(1)\right]^{2}} \frac{1}{t^{2}} \quad \text { for } t \gg T_{\{\alpha\}}^{*}, t_{\gamma} \\
\left\langle\left(\delta T_{\{\alpha\}} / T_{\{\alpha\}}^{*}\right)^{2}\right\rangle_{t} & =\left[2 /\left(h_{\{\alpha\}} \ln 2\right)^{2}\right]\left(1 / t^{2}\right) \\
\left\langle\delta T^{2}\right\rangle_{t} & =\frac{2\left(T_{(2)}^{*}\right)^{2}}{\left[\ln P_{(2) m}^{*}(1)\right]^{2}} \frac{1}{t^{2}} \text { for } t \gg T_{(2)}^{*} \\
\left\langle\delta \omega^{2}\right\rangle_{t} & =\left\{2 \omega_{0}^{2} /\left[\ln P_{(2) m}^{*}(1)\right]^{2}\right\}\left(1 / t^{2}\right)
\end{aligned}
$$

${ }^{1}$ This paper appeared in $J$. Stat. Phys. 11, 87 (1974).

${ }^{2}$ Central Research Laboratories, Nippon Electric Company, Ltd., Kawasaki, Japan. 
Equations (207) and (217) should read

$$
\lim _{n \rightarrow \infty}\left(n T_{i j} / \tilde{T}\right) \sim 1
$$

and

$$
Y \equiv \frac{1}{\mu(\Delta)} \frac{d}{d t} \int_{\bar{\Gamma}-\Delta} P\left(\Delta^{\prime}, d t\right) d \mu\left(\Delta^{\prime}\right)
$$

respectively. 